\title{
Diacronie
}

Studi di Storia Contemporanea

$N^{\circ} 18,2 \mid 2014$

Le esposizioni: propaganda e costruzione identitaria

\section{La Exposición Internacional de Barcelona en 1929 y su utilización propagandística}

La montaña de Montjuïc, espacio público de ocio, cultura y deporte Juan Antonio Simón

\section{(2) OpenEdition \\ Journals}

Edición electrónica

URL: http://journals.openedition.org/diacronie/1505

DOI: 10.4000/diacronie. 1505

ISSN: 2038-0925

Editor

Association culturelle Diacronie

Referencia electrónica

Juan Antonio Simón, « La Exposición Internacional de Barcelona en 1929 y su utilización

propagandística », Diacronie [En línea], № 18, 2 | 2014, documento 13, Puesto en línea el 01 junio 2014, consultado el 30 abril 2019. URL : http://journals.openedition.org/diacronie/1505 ; DOI : 10.4000/ diacronie. 1505 


\title{
Diacronie
}

\section{La Exposición Internacional de Barcelona en} 1929 y su utilización propagandística

\section{La montaña de Montjuïc, espacio público de ocio y deporte}

\author{
Juan Antonio SIMÓN *
}

La Exposición Internacional de Barcelona de 1929 se convirtió en el motor de modernización de la ciudad y, al mismo tiempo, en una herramienta de propaganda con la que se buscó la difusión de una nueva imagen de progreso y modernidad. Este artículo afronta el estudio de la transformación de la montaña de Montjuïc, lugar en el que se ubicó la Exposición, como nuevo espacio público de ocio, entretenimiento y deporte. Estrechamente relacionado con lo anterior, el deporte se convirtió en uno de los tres ámbitos en los que se desarrolló el certamen. Este texto analiza el papel del deporte en la Exposición como factor que impulsó la construcción de nuevas infraestructuras deportivas y la difusión de su prá ctica entre las clases populares de la sociedad catalana y española .

La Exposición Internacional de 1929 ha quedado presente en la memoria de los barceloneses por un legado imborrable: la rehabilitación de la montaña de Montjuïc y su transformación en el principal centro de cultura, ocio y entretenimiento de la ciudad. Este estudio pretende analizar cómo se utilizó la celebración de la Exposición Internacional, y en concreto la transformación de la propia montaña de Montjuïc, como un elemento ideal con el que conseguir transmitir al exterior la imagen de un país moderno y avanzado, que en pleno desarrollo de la sociedad de masas y en línea con la tendencia europea, había sido capaz de "conquistar" para sus ciudadanos un nuevo 
espacio público y convertirlo en el centro cultural y deportivo de la ciudad. Por este motivo, el estudio del papel que representó el deporte en el contenido de la Exposición de Barcelona es central en este artículo. Entendido como un factor de modernidad y progreso, las actividades físicas y deportivas disfrutaron de un gran protagonismo en la programación y tuvieron en el estadio olímpico su símbolo y principal legado histórico para la ciudad.

Pese a que fue elegido por los propios organizadores como uno de los tres grandes ámbitos en los que se desarrolló la Exposición, el estudio del impacto que tuvieron las múltiples actividades deportivas programadas, así como el discurso que desde la prensa se realizó sobre la importancia del fomento del deporte y la posterior herencia que estos factores dejaron en los hábitos de ocio y entretenimiento de la ciudadanía barcelonesa, han sido aspectos tristemente olvidados en los trabajos académicos publicados hasta el momento ${ }^{1}$. Este artículo pretende aportar una perspectiva nueva al estudio de las Exposiciones Internacionales con el deseo de que también pueda favorecer nuevos e interesantes debates.

\section{La Exposición de Barcelona de 1929}

Bajo la presidencia de honor del rey Alfonso XIII la Exposición Internacional de Barcelona de 1929 se desarrolló en tres grandes esferas temáticas: industria, arte y deporte. La primera de estas áreas buscó relanzar la industria española a nivel nacional e internacional, consiguiendo ayudar a la reactivación e impulso de la producción industrial. El segundo de los núcleos en los que se centró la Exposición fue el fomento del arte y la cultura tradicional española. La exposición de arte contemporáneo que se instaló en el Palacio de Bellas Artes, o el «primer museo artístico del mundo» que se organizó en el Palacio Nacional, son algunos ejemplos que subrayan la importancia que representó el arte y la cultura en el contenido de este certamen. En cambio, la cultura regional disfrutó en el Pueblo Español de un escenario creado a su medida².

Por último, el deporte, elemento intrínsecamente ligado a las Exposiciones Internacionales desde el nacimiento del Comité Olímpico Internacional en 1894 y las

\footnotetext{
${ }^{1}$ Una excepción a lo anterior la encontramos en FUNDACIÓN BARCELONA OLÍMPICA, L'esport a l'Exposició Internacional de Barcelona 1929, Barcelona, Fundació Barcelona Olímpica, 2012.

2 CASTRO LES, Vicente, «La Exposición Internacional de Barcelona», in Gran Vida, 1 Mayo 1929, pp. 161-164.
} 
primeras ediciones de los Juegos Olímpicos³, también disfrutó en Barcelona de un amplio programa de competiciones, con el que se quería difundir una imagen de modernidad y desarrollo al mismo tiempo que conseguir impulsar la difusión de la actividad física y deportiva entre la propia sociedad catalana y española. En relación con este último aspecto, el estadio olímpico construido en Montjuïc se convirtió en uno de los principales símbolos de la Exposición y, como también quiso destacar la prensa, en «el signo de la pujanza deportiva de Barcelona». Junto con el mencionado estadio, se crearon edificaciones como el Pueblo Español, el Pabellón de las Misiones, el Pabellón del Estado Español, además de otros quince edificios y pabellones de países como Italia, Alemania, Suecia o Francia, o de temáticas especificas como los centrados en las Artes Industriales y Aplicadas, Deportes, Confecciones, Electricidad y Metalurgia, Bellas Artes, Ciencias o Agricultura, logrando caracterizar a nivel arquitectónico la mayor transformación urbanística que había vivido la ciudad desde el inicio del siglo $\mathrm{XX}^{4}$.

La montaña de Montjuïc se convirtió en el lugar elegido para emplazar la Exposición. El reto de su urbanización supuso enfrentarse al problema que representaba la existencia en sus laderas de varias canteras de piedra, la ubicación del cementerio del Sudoeste así como la presencia de algunos barrios que se habían asentado en esta zona como eran Sant Beltrà, Santa Madrona y la Fransa ${ }^{5}$. Pese a todo, y recordando la mención que apareció publicada en un catalogo fotográfico de la época sobre la Exposición, «Barcelona, en pocos años, ha transformado parte de la histórica montaña de Montjuich en un frondoso parque de 200 hectáreas, con vistas a la ciudad y al mar, que sirve hoy de marco de insuperable belleza a su Exposición, la que, por su emplazamiento, su grandiosidad, su profusión de luz y juegos de aguas, se clasifica en primer lugar entre las celebradas en todo el orbe» ${ }^{6}$. El primero de los proyectos que impulsó el ayuntamiento de Barcelona para urbanizar el Parque de Montjuïc fue obra del arquitecto Josep Amargós en 1894. Su propósito era que las faldas de la montaña dejasen de ser un refugio «de gente de mal vivir», incidiendo en la importancia del ajardinamiento, la construcción de pequeños parques a lo largo de la montaña que la

\footnotetext{
3 MULLER, Norbert (editor), Pierre de Coubertin, 1863-1937: Olympism. Select writings, Lausanne, International Olympic Committee, 2000.

4 «Editorial», in Diario Oficial de la Exposición Internacional de Barcelona 1929, 9 Junio 1929, p. 9. Ver también: Exposición internacional. Barcelona: su significación y alcance, Barcelona, Seix y Barral, 1929; GRANDAS, M. Carmen, L'Exposició Internacional de Barcelona de 1929, Barcelona, Els Llibres de la Frontera, 1988; Cicerone de Barcelona-Exposición 1929, s.l., s.e., 1929; «La Exposición Internacional de Barcelona», in La Construcción Moderna, 15 Junio 1928, pp. 161-167 y 30 Junio 1928, pp. 177-180.

5 GRANDAS, M. Carmen, op. cit., p. 79.

${ }^{6}$ Exposición Internacional de Barcelona, 1929, Barcelona, s.n., 1929.
} 
convirtieran «en sitio de recreo y esparcimiento, como pocos existan en las poblaciones mediterráneas»7.

El proyecto de Amargós se recuperó cuando en 1916 la Junta Directiva de la Exposición decide contratar para dirigir el proyecto de urbanización y ajardinamiento de la montaña de Montjuïc al director de la conservación de los bosques de París, JeanClaude Nicolas Forestier. El ingeniero francés contó desde el primer momento con la ayuda inestimable del joven arquitecto Nicolau M. Rubio i Tudurí, quien mantuvo su fidelidad al estilo del proyecto que había iniciado Forestier ${ }^{8}$.

La organización de la Exposición Internacional en Barcelona provocó una radical transformación urbana. La propia prensa resaltó el gran número de reformas que se habían producido durante los últimos años en las calles y plazas de la ciudad, lo que provocaría que en nada tuviera «que envidiar, en cuanto a ornato público se refiere, a otras ciudades europeas y americanas, citadas siempre como modelo por los amantes de lo bello y de lo suntuoso»9. Desde lugares de gran simbolismo como la Plaza de Cataluña y la Plaza de España, pasando por la avenida Alfonso XIII, actual avenida Diagonal, la Rambla de Cataluña o el Paseo de Gracia, junto con innumerable calles y plazas fueron reformadas para dar a la ciudad el esplendor urbanístico que reclamaba la organización de la Exposición ${ }^{10}$. Al mismo tiempo, la reforma también provocó el aumento de la red de transportes públicos y la implementación del alumbrado en gran parte de las principales avenidas de la ciudad, uno de los grandes éxitos y reclamos propagandísticos que mostró al mundo Barcelona, un «milagro urbanístico» que tenía como principal responsable a la Exposición:

\footnotetext{
El milagro se debe a la Exposición Internacional que hoy permite ofrecer al Mundo un bello ejemplo de vitalidad de nuestra urbe, y un avance de lo que ésta llegará a ser en plazo breve. Y si, a las mejoras citadas, unimos las maravillas de Montjuich, cuyos jardines y avenidas subsistirán cuando la Exposición cierre sus puertas, y cuyos grandes Palacios, en su mayoría, quedarán en pie, para ser utilizados en la forma más conveniente, veremos que el balance que para Barcelona arroja ya hoy
}

\footnotetext{
7 AMARGÓS, José, «Urbanización de la montaña de Montjuich», en Arquitectura y Construcción, 21/1898, pp.10-15.

${ }^{8}$ GRANDAS, M. Carmen, op. cit., pp. 79-89.

9 BONO, Arturo F., «La transformación de nuestra ciudad», en Diario Oficial de la Exposición Internacional de Barcelona 1929, 22 Mayo 1929, p.14.

${ }^{10}$ Sobre la reforma urbana de Barcelona ver «Una gestión municipal afortunada», en Diario Oficial Exposición de Barcelona 1930, 18 de enero 1930.
} 
su magno certamen es altamente favorable y justifica de sobras todos los sacrificios $^{11}$.

A nivel propagandístico, tanto desde la perspectiva regional como a nivel nacional, la Exposición representaba un escenario perfecto para transmitir, tanto a nivel interno como hacia el exterior de las fronteras españolas, los progresos de esta sociedad. Rápidamente muchos autores se unieron en el elogio y la felicitación por lo que consideraban un gran éxito en la organización de la Exposición, que había provocado «iEl resurgimiento moral y material de la vieja España!» ${ }^{12}$. El final del siglo XIX había mostrado las carencias de un país que con la pérdida de sus últimas colonias había comprendido que estaba muy lejos de ser una potencia. Los teóricos del regeneracionismo que abogaron por una radical transformación de las nuevas generaciones para poder estar a la altura de nuestros vecinos europeos, parecía que habían encontrado respuesta en la imagen que transmitía décadas más tarde la Exposición de Barcelona:

Los países extraños, rendidos a la evidencia del hecho esplendoroso, hanse [sic.] visto obligados a reconocer, por sus órganos representativos y de Prensa, que España, en estos momentos para ella ciertamente históricos, ha culminado con la egregia pompa de una potencia de primer orden, en el trabajo y capacitación para su futuro desenvolvimiento ${ }^{13}$.

\section{El deporte en la Exposición Internacional de Barcelona}

$\mathrm{Al}$ final de la década de los veinte Barcelona asumió un gran reto para la ciudad con la organización de la Exposición International y, sobre todo, con la edificación de toda una serie de infraestructuras urbanas que ocuparían diferentes espacios en la montaña de Montjuïc. Desde el punto de vista de la importancia que se concedió a la actividad física y el deporte, la Exposición tendría dos líneas de actuación bien diferenciadas: por un lado, la construcción de nuevos espacios e instalaciones deportivas que facilitarían el impulso de la socialización de la cultura física en Barcelona; y al mismo tiempo, la implementación de un ambicioso programa de actividades deportivas que se

${ }_{11}$ A.R.D., «La Exposición de Barcelona y el mejoramiento urbano de la ciudad», in Diario Oficial de la Exposición Internacional de Barcelona 1929, 21 Julio 1929, pp. 25-26.

${ }^{12}$ GARCÍA, Ricardo, «España dentro y fuera de la Exposición», in Diario Oficial de la Exposición Internacional de Barcelona 1929, 30 Noviembre 1929.

13 Ibidem. 
convertirían en una magnifica herramienta publicitaría multiplicando el impacto internacional de este evento. Las magnificas competiciones programadas llenarían de contenido la inauguración de las modernas instalaciones que se inaugurarían durante estas semanas. El «afán de progreso» de la ciudad de Barcelona también tendría su reflejo en el «Núcleo Deportivo» de la Exposición:

El auge que los deportes han adquirido en todos los países, la importancia creciente que se les asigna en los modernos sistemas pedagógicos y el movimiento industrial y turístico que en torno a ellos se viene desarrollando, justifica plenamente que se consagre a esta clase de manifestaciones un Palacio especial, además del anchuroso terreno que abarca el Estadio ${ }^{14}$.

El tercer pilar conceptual de la Exposición también buscó incorporar Barcelona «al dinamismo de la Europa moderna”. Deporte y desarrollo urbano fueron de la mano en los años veinte, por lo que no se escatimó esfuerzos para crear un programa deportivo lo más amplio posible que permitiera ofrecer una imagen de desarrollo y progreso. La prensa no dudó en destacar la importancia que tendría el programa deportivo de la Exposición para lograr la deseada difusión de nuevos hábitos saludables e higiénicos entre las clases populares. Jóvenes, ancianos y mujeres debían ser conscientes de la importancia que tenía la intensificación del ejercicio físico, y la Exposición debía ser la herramienta perfecta para transmitirlo:

Si el rastro de la Exposición-en este aspecto y en todos- se traduce en una intensificación del ejercicio de la cultura física en las escuelas e incita a la muchedumbre sedentaria de nuestras innumerables oficinas comerciales a buscar en la gimnasia la compensación que ha de convertirse en salud para el cuerpo, ¿̇a qué más podemos aspirarir?

A finales de 1928 se crea el Comité Deportivo de la Exposición Internacional. Este organismo estuvo presidido por Mariano de Foronda, marqués de Foronda, como director de la Exposición Internacional, junto con reconocidos nombres del deporte en la sociedad catalana como el capitán Castell; Josep Mesalles Estivil, miembro de la Confederación Deportiva de Catalunya y secretario del Comité Olímpico Español (COE); y Narcís Masferrer, periodista e incansable propagador del deporte en Cataluña

${ }^{14}$ Exposición internacional. Barcelona: su significación y alcance, cit., p. 35.

15 VINARDELL, Santiago, «Los Deportes en la Exposición», in Diario Oficial de la Exposición Internacional de Barcelona 1929, 5 Mayo 1929, p. 11. 
y también representante del máximo organismo olímpico español. A finales del mes de abril de 1929 el Comité Deportivo publicó un ambicioso programa de actividades deportivas, en el que se incluyeron 26 diferentes especialidades que irían desde la celebración de pruebas de automovilismo y motorismo, pasando por competiciones de atletismo, aviación, fútbol, esgrima, boxeo, ciclismo o natación. Aunque algunas de las actividades no estaban todavía cerradas, el «Diario Oficial» de la Exposición publicó dicho programa agradeciendo a los organizadores su empeño en el impulso del deporte, y la concesión al Comité Deportivo de «un amplio crédito e ilimitado margen de confianza», para que pudieran «confeccionar un programa de juegos deportivos digno de Barcelona y de su Exposición Internacional» ${ }^{16}$.

El programa de actividades deportivas de la Exposición también permitió descubrir la importancia que otros países concedían a estas actividades como elemento clave en el encuadramiento político y social de sus ciudadanos. Un ejemplo de lo anterior lo encontramos en las noticas relacionadas con el «Torneo Latino» de boxeo amateur, celebrado dentro de la programación de la Exposición y que enfrentó a boxeadores de España, Bélgica, Francia e Italia. La potencia atlética de los deportistas transalpinos era muy conocida en Europa desde la llegada de Mussolini al poder en 1922. Supeditado a los interese políticos, el deporte en Italia durante la dictadura se convirtió en una herramienta de la propaganda fascista ${ }^{17}$. El director técnico del equipo italiano, el señor Volpi, no desaprovechó la oportunidad que le brindaba la prensa española para elogiar la importancia que el duce concedía a la formación deportiva de sus ciudadanos:

La importancia que el Gobierno italiano le da a los deportes, tomándolos bajo su protección, obliga a todo italiano que tiene el honor de defender el prestigio de su país, en justa correspondencia, a agotar todas sus reservas físicas y morales antes de considerarse vencido. Y para que pueda competir sin desventaja alguna, se le

\footnotetext{
${ }^{16}$ LÓPEZ-MARQUES, A., «Los Deportes en la Exposición», in Diario Oficial de la Exposición Internacional de Barcelona 1929, 21 Abril 1929, pp. 26-27. Destacar que aprovechando la Exposición de Barcelona, la FIFA decidió organizar en esta ciudad su asamblea anual con la que se celebraba el 25 aniversario de su fundación: «La vida deportiva barcelonesa», in Diario Oficial de la Exposición Internacional de Barcelona 1929, 21 Abril 1929, p. 39. «El Congreso de la Federación Internacional de Fútbol», in Diario Oficial de la Exposición Internacional de Barcelona 1929, 5 Mayo 1929.

${ }_{17}$ Ver algunos ejemplos en FABRIZIO, Felicie, Sport e fascismo: La politica sportive del regime 1924-1936, Rimini-Firenze, Guaraldi Editore, 1976; GRAZIA, Victoria de, Consenso e cultura di massa nell'Italia fascista: l'organizzazione del dopolavoro, Roma, Laterza, 1981; MARTIN, Simon, Football and Fascism: The National Game Under Mussolini, Oxford, Berg, 2004; PAPA, Antonio, PANICO, Guido, Storia sociale del calcio in Italia: Dai club dei pioneri alla nazione sportiva (1887-1945), Bologna, Il Mulino, 1993.
} 
conceden cuantas facilidades le son precisas para el entrenamiento, por parte del

Estado y de los particulares ${ }^{18}$.

\section{El Estadio de Montjuïc símbolo de la Exposición Internacional de Barcelona}

A requerimiento de la Confederación Deportiva de Cataluña y dado las carencias que presentaba el antiguo estadio de la Fuxarda, instalación construida en 1921 con la intención de ser el epicentro de la candidatura de Barcelona para los Juegos Olímpicos de 1924, el marqués de Foronda decidió que en la parte alta del Parque de Montjuïc, concretamente en la conocida como Plaza de San Antonio junto a la gran avenida central del Marqués de Comillas, se ubicase el nuevo estadio que albergaría las principales competiciones deportivas. Esta infraestructura deportiva se convertiría en el símbolo de la Exposición, ejemplo de innovación arquitectónica y desarrollo tecnológico. Es indudable que en la mente de los organizadores de la Exposición y de los propios arquitectos estaba muy presente el impacto que en 1923 había supuesto la inauguración del estadio de Wembley en Londres ${ }^{19}$.

El estadio de Montjuïc se convertiría en el segundo de mayor capacidad en Europa después precisamente del estadio londinense. El proyecto del arquitecto Pedro Domenech i Roura abarcaría 45.225 metros cuadrados, con unas gradas capaces de albergar 60.00o espectadores, cifra que superaba la capacidad del moderno estadio de Colombes en París, quedando únicamente por detrás de los cien mil espectadores que podían acoger las gradas de Wembley. El terreno de juego era de hierba, con unas medidas de 105 de largo por 70 de ancho y dejando espacio suficiente para las instalaciones de atletismo. En la tribuna principal se instalaron dos amplios halls a los que se podría «ingresar desde el exterior por medio de unos pórticos que conducirían también a la parte baja de las graderías y a los locales del bar y restaurante ${ }^{20}$. La nueva construcción también tenía previsto convertirse en la sede social del COE y de la Real Federación Española de Fútbol (RFEF), destinando para este fin diferentes despachos y salas de juntas en su interior. Del mismo modo, las instalaciones contarían con dos salas de prensa con «aparatos para las comunicaciones telefónicas y telegráficas y

\footnotetext{
${ }^{18}$ «Los Deportes en la Exposición. El gran Torneo Latino de boxeo amateur», in La Vanguardia, 11 Julio 1929, p.13.

19 Un ejemplo del impacto de la inauguración del estadio de Wembley en la prensa española: ALCARAZ, E., "La final de la "Copa Inglesa"», en Madrid-Sport, 10 Mayo 1923, pp. 12-13. 20 «El Estadio de la Exposición», in Diario Oficial de la Exposición Internacional de Barcelona 1929, 21 Abril 1929, pp. 32-33.
} 
demás elementos que necesitan los periodistas durante las largas fiestas deportivas» ${ }^{21}$. Dos vestuarios para 25 personas cada uno, con duchas, sala de masaje y piscina, se unían a los servicios que ofrecía el estadio, a lo que se añadían varios pabellones anexos «destinados a boxeo, gimnasia y esgrima, y una gran piscina para juegos acuáticos. Junto al palacio de deportes habrá una pista para “tennis”, y se reservará un lugar para los pabellones de las Sociedades deportivas» ${ }^{22}$.

El propio Narciso Masferrer publicó un artículo para «La Vanguardia» en enero de 1929 analizando la importancia de dicho proyecto:

\begin{abstract}
¡Vamos a movilizar el ejército deportivo! Los que lo practican deberán ir al Estadio a rendir sus máximos esfuerzos, bien entrenados, bien alecionados [sic.] y dispuestos; quienes sientan admiración y devoción por el sport, deberán acudir en proporción tal y tan grande que se den siempre la sensación de que al construirse el Estadio, se nos brindó la ocasión de exteriorizar nuestros entusiasmos y nuestros amores por la portentosa obra de cultura, de fraternidad y amor que simboliza el sport en todos sus infinitos aspectos.

Hagámonos dignos, en ocasión de la Exposición de 1929, de que el Estadio de Montjuich se considere el marco más adecuarlo para los Juegos Olímpicos de 1936, suprema y última aspiración del que suscribe ${ }^{23}$.
\end{abstract}

En cuanto a su estilo arquitectónico, Buenaventura Basegoda, profesor de la escuela de arquitectura de Barcelona, destacaba lo acertado de haber dado un «sello clásico y monumental» al edificio. La estructura central se caracterizaría por su «corte severo y majestuoso», destacando la parte correspondiente al servicio de la Majestad real, a cuyo centro acompañan dos galerías laterales arqueadas con amplias terrazas, que constituyen espléndido mirador sobre la grandiosa avenida» ${ }^{24}$. Como última mención respecto a las características que mostraba el estadio más moderno del país, señalar que la monumental fachada principal tenía una cúpula en donde destacaban los «Jinetes haciendo el saludo olímpico», dos grandes esculturas ecuestres en bronce de Pablo Gargallo25. Los progresos en la construcción del que se consideró el «segundo Estadio de Europa, y al que en disposición técnica ningún otro puede superar», crearon

${ }^{21}$ «El stadium de la Exposición de Barcelona», in La Construcción Moderna, 15 Abril 1927, p. 111.

${ }^{22}$ Ibidem. Respecto a las características del estadio ver también Exposición internacional. Barcelona: su significación y alcance, cit., p. 35-37

23 MASFERRER, Narciso, «Lo que será el Estadio», in La Vanguardia, 1 Enero 1929, pp. 22-23.

24 X. X., «El estadio de Montjuich, de Barcelona», in La Construcción Moderna, 30 Mayo 1929, pp. 150-154.

25 GRANDAS, M. Carmen, op. cit., 1988, pp. 79-89. 
una enorme expectación que iría aumentando según se aproximaba la fecha de su inauguración.

Como no podía ser de otro forma ante la interés que se creó durante los meses previos, uno de los acontecimientos más esperados de la Exposición fue la inauguración del estadio de Montjuïc. Al acto celebrado el 20 de mayo de 1929 acudieron las más importantes personalidades del momento, entre las que podemos destacar el propio rey Alfonso XIII; el conde Baillet Latour, como presidente del COI; el barón de Güell, miembro español del COI; y el marqués de Polignac, representante del Comité Olímpico francés, junto con un buen número de representantes políticos y deportivos catalanes. Las tribunas del estadio se llenaron con 65.000 espectadores, y la organización tuvo que poner autocares para poder llevar al público desde la Plaza de España hasta el estadio. La intención inicial del Comité Deportivo era inaugurar el estadio con el encuentro de fútbol entre España e Inglaterra, pero dicho partido se terminó celebrando una semana antes en el estadio Metropolitano de Madrid. Rápidamente creció la preocupación entre los organizadores de la Exposición ante el temor a que uno de los acontecimientos más esperados no pudiera ofrecer un festival deportivo de un prestigio internacional. Después de barajar la posibilidad de organizar un encuentro entre «un equipo representativo de Barcelona y el de Viena o Torino» ${ }^{26}$, se consiguió que el actual campeón de la Copa inglesa, el Bolton Wanderers, acudiese a Barcelona para disputar un partido contra una selección de futbolistas catalanes de los equipos del FC Barcelona, Español y Europa.

Ese mismo día, también se celebraría un encuentro de rugby entre las selecciones de España e Italia, y por último, se disputarían varias carreras atléticas de 100 y 200 metros. El marqués de Foronda había conseguido que pese a no poder contar con el atractivo que habría supuesto el partido de fútbol entre España e Inglaterra, la inauguración del estadio iba a tener la resonancia internacional que merecía este acto. El Comité Deportivo llegó a un acuerdo con el Bolton Wanders ofreciéndole un contrato de 2.250 libras, alrededor de 76.000 pesetas por la disputa del partido ${ }^{27}$. La entrada general costaba 3 pesetas y un palco principal 100, incluyendo en el precio la visita al recinto de la Exposición. La recaudación alcanzó las 415.00o pesetas, una cifra récord en cualquier evento deportivo celebrado hasta la fecha en España. Los aficionados terminaron disfrutaron con la victoria de la selección española de rugby por 9 a o, así como con el triunfo del combinado catalán por 4-o frente al equipo inglés; poniendo un

26 «La vida deportiva barcelonesa», in Diario Oficial de la Exposición Internacional de Barcelona 1929, 21 Abril 1929, p. 39.

27 «El Estadio de Montjuich», in Diario Oficial de la Exposición Internacional de Barcelona 1929, 5 Mayo 1929, p. 22. 
gran colofón a una jornada histórica tanto para el deporte como para la propia Exposición ${ }^{28}$.

La construcción del estadio de Montjuïc y el extenso programa deportivo de la Exposición también buscaba mostrar la capacidad de Barcelona para poder organizar la próxima edición de los Juegos Olímpicos ${ }^{29}$.Por este motivo, la inauguración se aprovechó para mostrar al presidente del COI y padrino del estadio de Montjuïc, Baillet Latour, el interés de Barcelona en presentar su candidatura para la futura olimpiada de 1936. En el banquete con el que la Confederación Deportiva de Cataluña y el COE obsequiaron a Baillet Latour, éste dejó clara su opinión sobre los trabajos que se habían realizado: «Me ha impresionado grandemente. Diga usted que España puede sentirse orgullosa de la alta cultura deportiva de su pueblo que ha demostrado hoy ser digno de la capacidad de un templo deportivo tan grande como este» ${ }^{\circ}$.

Durante los meses posteriores se celebraron en el estadio un amplio número de competiciones deportivas, logrando de esta forma dar continuidad al éxito que había generado la inauguración de Montjuïc. Durante los meses en los que se desarrolló la Exposición un amplio número de encuentros de fútbol se realizaron en el estadio, sirviendo como un magnifico reclamo para atraer gente a las instalaciones de la muestra. El Comité Deportivo llegó a un acuerdo con los diferentes clubes para que sus socios pudieran asistir al estadio y visitar la Exposición sin tener que pagar nada ${ }^{31}$. El propio presidente del FC Barcelona, Tomás Rosés, declaró a la prensa que la celebración de los partidos del Campeonato de Liga en el estadio sería un éxito, y que su club había "procurado dar todas las facilidades posibles para que los encuentros puedan celebrarse en el recinto Olímpico. Debemos cooperar todos a la obra magna del Estadio y asegurar sus éxitos». Para concluir, mencionó que la Exposición le había parecido «un éxito rotundo. Deje que vayan a los países los primeros extranjeros que la han visitado y verá usted cómo afluye a Barcelona una riada humana»32.

\footnotetext{
${ }^{28}$ «Bajo la presidencia de los Reyes y con asistencia de setenta mil personas se inauguró ayer el estadio de Montjuich», en Diario Oficial de la Exposición Internacional de Barcelona 1929, 21 Mayo 1929, pp.16-18. "Inauguración del nuevo Estadio de Barcelona», in Gran Vida, 1 Mayo 1929, pp. 165-167. «La Barcelona deportiva cuenta con uno de los mejores Estadios del mundo», en Stadium, 1 Junio 1929, pp. 16-20.

${ }^{29}$ «El Estadio de la Exposición», en Diario Oficial de la Exposición Internacional de Barcelona 1929, 21 Abril 1929, p.32. Discursos similares aparecen en Cicerone de Barcelona-Exposición 1929, cit., 1929, p. 31. OLLE BERTRAN, A., «Los Deportes en la Exposición», in Diario Oficial de la Exposición Internacional de Barcelona 1929, 12 Mayo 1929, p.21.

30 «Excmo. Sr. Conde de Baillet del C.O.I., (Padrino del Estadio de Montjuich)», in Diario Oficial de la Exposición Internacional de Barcelona 1929, 21 Mayo 1929, p.18.

${ }^{31}$ CIERVO, Joaquín, «Los Deportes en la Exposición», in Diario Oficial de la Exposición Internacional de Barcelona 1929, 24 Mayo 1929, p. 10.

32 OLLE BERTRAN, A., «Los Deportes en la Exposición», in Diario Oficial de la Exposición Internacional de Barcelona 1929, 24 Mayo 1929, p. 17.
} 
El ciclismo también tuvo la posibilidad de presentarse dentro en la Exposición, gracias a la organización de varias carreras entre las que destacó por su importancia internacional la Toulouse-Barcelona, tradicionalmente conocida como el «Criterium Cyclista du Midi», que en esta edición impulsaron los periódicos españoles «El Día Gráfico» y la «Gaceta Deportiva», junto con el periódico francés «La Dépêche». La última etapa de esta prueba ciclista por etapas concluiría dentro del estadio de Montjuïc33. Coincidiendo con el final de la mencionada prueba también se celebraron en el estadio un festival de atletismo entre España e Italia, y varias competiciones de «dirt-track», carreras de motos aprovechando la pista de ceniza de atletismo. Los precios populares (las entradas generales costaban una peseta) buscaban dar mayor popularidad a estos festivales internacionales que no tenían en nuestro país el atractivo que disfrutaba el fútbol. Pese a todo, el principal objetivo de estos festivales deportivos que se organizaron durante la Exposición, fue el mostrar a través del deporte una imagen de progreso y modernidad, que contrastaba con la realidad que se vivía en gran parte del territorio español:

Cuando al pasar la frontera los corredores y seguidores extranjeros se hacían lenguas de la facilidad inmejorable con que unos y otros la atravesaban, pudieron ya apercibirse de que entraban en un país que les acogía con los brazos abiertos. [...] No han sido los españoles los vencedores de la gran carrera, pero lo han sido triunfando en el corazón y la voluntad de todos. Hemos demostrado nuestra superioridad moral por nuestro espíritu noble y hospitalario. Y éste sí que es éxito absoluto34.

\section{El ocio y el deporte conquistan la montaña de Montjuïc}

Como ya se ha mencionado previamente, el deporte fue una de las tres grandes áreas temáticas en las que se desarrolló el contenido de la Exposición. Uno de los principales objetivos de los organizadores era conseguir que la montaña de Montjuïc se convirtiese en el principal centro de ocio de los barceloneses. Llegados a este punto, es necesario recordar que el estadio no fue la única infraestructura deportiva que se construyó en estos meses. Junto con los amplios y hermosos jardines y parques de la

\footnotetext{
${ }^{33}$ LÓPEZ-MARQUES, A., «Los Deportes en la Exposición», in Diario Oficial de la Exposición Internacional de Barcelona 1929, 30 Junio 1929, p. 19.

34 BORJA, «Montjuich centro internacional del deporte», in Diario Oficial de la Exposición Internacional de Barcelona 1929, 22 Mayo 1929, pp. 21-22. Sobre las pruebas de «dirt-track» se pueden consultar entre otras muchas noticias en la misma publicación «Las emocionantes carreras de dirt-track en el Estadio», 7 Septiembre 1929, p. 31.
} 
Exposición, las múltiples fuentes con sus espectaculares juegos de agua y el atractivo del Parque de Atracciones, Montjuïc ofrecía a sus visitantes una moderna piscina municipal y unas instalaciones para la práctica del tenis. Además de esto, la organización también programó diferentes actos y festivales deportivos en otros tres recintos: el majestuoso Palacio Nacional, donde se acogerían diferentes competiciones de esgrima y boxeo; el Polígono, en el que se celebrarían concursos de tiro; así como el Teatro Griego, instalación al aire libre inspirado en los antiguos teatros griegos, en la que se presenciaron diferentes competiciones de lucha35. Por último, añadir que el Palacio del Material Deportivo, diseñado por el arquitecto Antonio Sardá y que posteriormente pasó a denominarse Palacio de la Química, se proyectó inicialmente para acoger una gran exposición de materia deportivo aunque finalmente se decidió dedicarla a la industria química ${ }^{36}$. Los organizadores creyeron en un primer momento que no era suficiente con «la exhibición fugaz de las diversas manifestaciones deportivas si no va acompañada, para ser fructífera, de los necesarios valores documentales»; por lo que trataron de que la práctica deportiva no se quedase en una moda pasajera, mostrando a los visitantes «la pujanza actual de el [sic.] deporte en aquellos pueblos que figuran a la cabeza de la moderna civilización»37.

Cerca del Pueblo Español se ubicaron las pistas de tenis con las que el Comité Deportivo quería dar cabida a otras especialidades deportivas que no podían encontrar su espacio en el estadio. Junto al pequeño edificio o «chalet» que servía como vestuario se construyó la pista central rodeada de unas tribunas con capacidad para 3.000 espectadores. En un entorno natural, rodeado por un bosque, se decidió construir una segunda pista de menor tamaño para los entrenamientos. «El pequeño Wimbledon» como lo denominó la prensa, había sido diseñado por la firma londinense «En Tout Cas» y se convirtió en la mejor instalación para la práctica del «lawn-tennis» de toda España:

No hay lugar en la Exposición toda, mejor situado y desde el que disfrute de mayor sensación de hallarse muy apartado de la gran ciudad y aun de la Exposición misma. El bosque que circunda las instalaciones de tennis con su fragancia silvestre hacen de aquéllas algo recogido del mundanal ruido y es, sin embargo, en medio de este bosquecillo donde veremos congregada la mejor sociedad barcelonesa que

35 LÓPEZ-MARQUES, A., «La importancia deportiva del certamen», in Diario Oficial de la Exposición Internacional de Barcelona 1929, 22 Mayo 1929, p. 23.

36 GRANDAS, M. Carmen, op. cit., pp. 164-165; Exposición internacional. Barcelona: su significación y alcance, cit., p. 21.

37 VINARDELL, Santiago, «Los Deportes en la Exposición», in Diario Oficial de la Exposición Internacional de Barcelona 1929, 5 Mayo 1929, p. 11. 
asistirá a contemplar, como lo efectúa siempre, la lucha de jugadores de gran categoría cual si fuera un pequeño Wimbledon ${ }^{3}$.

Para su inauguración se concertó un campeonato que enfrentó a varios tenistas españoles con las mejores figuras del tenis norteamericano, entre los que se encontraban Allison y Van Ryn, los últimos ganadores de la prueba de dobles en Wimbledon39. Pese a todo, el tenis en España era un deporte que sólo practica a finales de los años treinta una reducida elite social, lo que provocó que la inauguración no despertase el atractivo suficiente entre el público: «el magnífico "court” de Montjuich no tuvo la solemne inauguración que merecía. Esperemos que los matchs de hoy o acaso los de septiembre, que aun podrían compensarnos de esta semi-decepción de ayer»40.

Cerca del estadio, en la Avenida Miramar, se ubicó otra de las más importantes instalaciones deportivas que se construyeron coincidiendo con la celebración de la Exposición: la piscina municipal. Pese a los retrasos que sufrió su edificación, concluyéndose más tarde que el estadio y las pistas de tenis, esta infraestructura superaba con creces la capacidad y condiciones de cualquier otra piscina existente en el país. La reiterada reclamación que durante los años previos se había realizado al ayuntamiento desde diferentes sectores de la opinión pública, se había convertido en realidad. La Exposición se apuntaba un nuevo éxito deportivo:

Desde el punto de vista deportivo y como complemento al vasto plan de la Exposición, la Piscina es un éxito más que añadir a los incalculables que viene obteniendo nuestro gran Certamen. Con ella se aumenta el prestigio de nuestra deportividad y se aumenta en una más las bellas construcciones de Montjuich41.

La construcción de la piscina se insertaba, como las anteriores infraestructuras deportivas realizadas para la Exposición, dentro de una clara estrategia de impulso y fomento de la verdadera democratización o socialización de la actividad deportiva entre las clases populares. Con estos proyectos se buscaba «aumentar en proporciones insospechadas el ambiente a la admiración y práctica de la natación», tanto el estadio,

${ }^{8}$ TEY, Manuel, «Los Deportes en la Exposición», in La Vanguardia, 5 Julio 1929, p.16.

39 A., «Los Deportes en la Exposición. El match de tennis Estados unidos-España», in La Vanguardia, 16 Julio 1929, pp. 19-20.

40 M.C., ««Los Deportes en la Exposición. El match de tennis Estados unidos-España», in $\mathrm{La}$ Vanguardia, 14 Julio 1929, p. 16.

${ }_{41}$ BORJA, «La piscina de Montjuich», in Diario Oficial de la Exposición Internacional de Barcelona 1929, 4 Agosto 1929, p. 21; PELLICER, Emilio, «El aspecto utilitario social de la natación», in La Vanguardia, 17 Agosto 1929, p. 12. 
como las pistas de tenis y la piscina se convertían para los organizadores en símbolos de «la definitiva convicción de un pueblo que crece en deportividad rapidísimamente, pero que une a su desarrollo una inteligencia adecuada con la que demuestra una orientación noble y sana que puede ponerla pronto al nivel de la nación más adelantada»42.

\section{Conclusiones}

La Exposición Internacional de Barcelona de 1929 se convirtió en un elemento catalizador para la ciudad, tanto desde ámbitos que hacían referencia de forma directa con la cultura, la industria y la economía, o también en relación con aspectos referidos a la arquitectura, la ideología, la propaganda y el urbanismo. Junto a los factores anteriores, este artículo ha demostrado la importancia que representó el deporte en el programa de la Exposición, convirtiéndose en un escaparate ideal para mostrar al mundo la preparación de Barcelona a la hora de organizar grandes eventos internacionales. Tanto los organizadores como las autoridades políticas y los propios medios de comunicación fueron conscientes del gran desafío que suponía para la ciudad el albergar la Exposición. Desde el diario catalán «La Vanguardia» se recordaba meses antes de su inauguración a los clubes, federaciones y atletas que «vamos a mostrarnos frente al mundo entero», y que no sólo estaba en juego el prestigio y la imagen de la ciudad y el país, sino que era necesario «mostrarse esplendorosamente» para conseguir vencer en la «apuesta que está en pie de si, a juicio del Congreso Olímpico de Berlín (1931), Barcelona es digna o no lo es de que le sean concedidos los juegos de la XIII Olimpiada»43.

Como ocurrió con la industria y el arte, el deporte también fue instrumentalizado por las autoridades políticas a nivel local y nacional así como por el propio comité organizador. Un claro ejemplo de lo anterior fue la construcción de avanzadas infraestructuras deportivas como la piscina, las pistas de tenis y, sobre todo, el estadio olímpico, que permitieron a Barcelona situarse al mismo nivel que cualquier otra ciudad europea, convirtiéndose al mismo tiempo en un elemento de orgullo para la dictadura de Primo de Rivera. Al mismo tiempo, la Exposición preparó un amplísimo programa de actividades deportivas entre las que tuvieron espacio especialidades que irían desde encuentros de fútbol, hockey, beisbol y rugby; pasando por festivales de

42 Ibidem.

43 «Los Deportes en la Exposición. Programas de fiestas», in La Vanguardia, 26 Abril 1929, p. 14 . 
atletismo, carreras ciclistas, combates de boxeo; o competiciones de motociclismo, automovilismo y deporte aéreo. En definitiva, una apuesta deportiva que hemos visto que pretendía conseguir la verdadera difusión de la actividad física y deportiva entre la sociedad barcelonesa y de los valores que iban implícitos en "el sport".

El proyecto deportivo de la Exposición consiguió dejar una huella que ha permanecido hasta nuestros días en Barcelona. Finalmente la candidatura olímpica de Berlín se impuso a Barcelona, pero el legado urbanístico, cultural y deportivo que representó para la ciudad la transformación de la montaña de Montjuïc que había impulsado la organización de la Exposición, permitió desarrollar las bases que en 1992 lograron convertir el anhelo en realidad. 


\section{* El autor}

Juan Antonio Simón es licenciado en historia por la Universidad Compluten se de Madrid. Doctor en Humanidades por la Universidad Carlos III de Madrid. Desde el 2006 ha trabajado en el Departamento de Humanidades de la Universidad Carlo s III de Madrid, en el Centro de Estudios Olímpicos de la Universidad Autónoma de Ba rcelona (CEO-UAB) y en la actualidad es profesor de historia del deporte en la Universidad Europea de Madrid (UEM). Ha publicado: SIMON, Juan Antonio, BONDINI, Gianni, Il suo nome è Pablito: l'Italia s’innamora degli azzurri e la Spagna scopre la democrazia, Roma, Absolutely Free Editore, 2014.

URL: < http://www.studistorici.com/progett/autori/\#Simon >

\section{Per citare questo articolo:}

SIMÓN, Juan Antonio, «La Exposición Internacional de Barcelona en 1929 y su utilización propagandística. La montaña de Montjuïc, espacio público de ocio y deporte», Diacronie. Studi di Storia Contemporanea : Le esposizioni: propaganda e costruzione identitaria, 29/6/2014,

URL: < http://www.studistorici.com/2014/6/29/simon_numero_18/ >

Diacronie Studi di Storia Contemporanea $\beta$ www.diacronie.it

Risorsa digitale indipendente a carattere storiografico. Uscita trimestrale. redazione.diacronie@hotmail.it

Comitato di redazione: Jacopo Bassi - Luca Bufarale - Elisa Grandi - Deborah Paci - Fausto Pietrancosta - Matteo Tomasoni - Luca Zuccolo

iritti: gli articoli di Diacronie. Studi di Storia Contemporanea sono pubblicati sotto licenza Creative Commons 2.5. Possono essere riprodotti a patto di non modificarne i contenuti e di non usarli per fini commerciali. La citazione di estratti è comunque sempre autorizzata, nei limiti previsti dalla legge. 